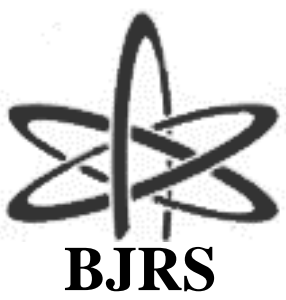

BRAZILIAN JOURNAL
OF
RADIATION SCIENCES
03-1A (2015) 01-10

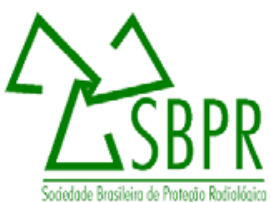

\title{
Análise fatorial de um feixe de prótons
}

\author{
A. R. S. Sena ${ }^{\text {a; J. W. Vieirab; F. R. A. Lima }}$ \\ ${ }^{a}$ DEN, UFPE, 50740-545, Recife-PE, Brasil \\ arssena@gmail.com \\ ${ }^{b}$ IFPE, 50740-545, Recife-PE, Brasil \\ jose.wilson59@uol.com.br \\ ${ }^{c}$ CRCN, CNEN, 50740-545, Recife-PE, Brasil \\ falima@cnen.gov.br
}

\begin{abstract}
Proton therapy is a technique for treating neoplasms using heavy particles that have a unique feature in the energy transfer process in the material means; the beam has a very well defined shape with well defined range for each rest mass and kinetic energy. In recent years, this technique has been widely used, especially in the U.S., the seventies until March 2014 there were more than 100,000 applications worldwide. This study aimed to study the relevant characteristics of a beam of charged heavy ions, specifically proton analyze which parameters are critical in the deposited dose, which can be relegated to the background and which can be neglected. With $2^{\mathrm{k}}$ factorial analysis, which proposes tests defined to determine the effects of certain parameters on the final result. Defining a set of input variables that are controllable and can interfere with the results of dose are, Energy Beam, Distance from Source Density Target, Target Temperature and Atmospheric Pressure. What variables have influence and their combinations cause changes in the average result of dose deposited by a computational voxel phantom. With the set of GEANT4 codes was simulated a mono-energetic protons entering a mathematical phantom voxel in different situations. Showed that the five variables, two are the most significant to the results of the dose, and Energy Density, and another being an inversely proportional.
\end{abstract}

Keywords:

Factor Analysis, Proton Therapy, Heavy Ion Radiotherapy. 


\section{INTRODUÇÃO}

O interesse e a necessidade de desenvolver e melhorar processos de estudos, tendo como um dos objetivos a melhoria da qualidade da dose sobre um órgão, suscitou a utilização de técnica estatística, planejamento ou análise de experimentos.

Ao planejar um experimento é possível se confrontar com algumas características finas do processo, que dificulta a execução experiências detrimento a saúde “in vivo" e gastos com tempo muito longo para executar um ensaio. Experimentos são realizados com a finalidade de aumentar a compreensão sobre um processo, definido por Montgomery[1] como sendo um teste, ou série de testes, em que mudanças propositais alterem o comportamento do processo. Essas mudanças são identificadas e quais fatores a influenciaram de forma mais significativa. Daí a importância de saber quais variáveis de entrada controláveis são mais importante(s) no resultado do processo, alterando a saída em função da entrada e de suas variáveis de entrada.

Com o conjunto de códigos GEANT4[2] que fora criado para simular cenários de fonte, ambiente, feixe de partículas, geometria com matéria equivalente ao tecido humano e as interações características de uma partícula pesada carregada no meio de interesse. Com o intuito de obter a energia depositada em um volume. Nesse processo quais variáveis tem influência na forma como o próton deposita sua energia no ponto de máxima profundidade.

Para a pesquisa desenvolvida definiu-se como objetivo geral, como se comportamento da dose por um feixe de prótons, com o foco no feixe de próton terapia. O objetivo especifico é expor conceitos básicos de experimentação por partículas carregadas, expor quais variáveis tem peso significativo no processo de deposição de energia no volume de interesse. 


\section{MATERIAIS E MÉTODOS}

Experimento tem o objetivo de compreender o processo, de que forma ele é influenciado. Quais variáveis de entrada controláveis são mais importantes no processo, alterando a saída em função de suas variáveis de entrada com esquematizado na Fig 1. As variáveis são ditas controláveis se suas entradas, denominada de níveis, forem passiveis de controle antes do início do processo.

Figura 1: Representação de um modelo de processo.

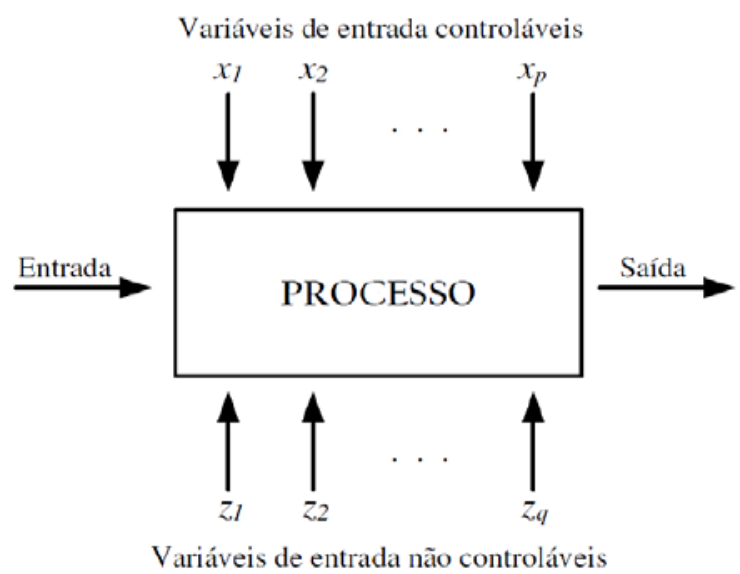

\subsection{EXPERIMENTO $2^{\mathrm{K}}$ DO FEIXE DE PRÓTONS}

No experimento fatorial obtém-se informações para uma região experimental maior do que cada efeito fatorial, pois é calculado sobre todas as combinações possíveis dos níveis dos outros fatores.

No planejamento de $k$ fatores onde ( $\quad$ ), cada um com dois níveis, que podem ser quantitativos - como valores de temperatura, pressão, tempo, ou qualitativos - tipo de máquina e/ou operador. Os níveis podem ser nomeados para distingui-los, de "baixo" e "alto", e "ausente" e "presente" e representados por códigos "0" e "1", ou "-" e "+", respectivamente. 


\subsubsection{VARIÁVEIS ANALISADAS}

Na avaliação de quais parâmetros são realmente fundamentais no estudo da dose do feixe na terapia por prótons, dos possíveis parâmetros de entrada, que alteraram a dose, foram escolhidos os que tivessem possibilidade de maior influência, segundo a teoria, fora escolhido os seguintes: Distância; Energia do feixe; Temperatura; Pressão atmosférica; Densidade do alvo, pois cada uma das variáveis tem a proposta de se relacionar com a dose de forma exclusiva. Para aumentar a visualização do efeito da interação extrapolou-se os valores a serem trabalhados, como por exemplo a temperatura, no lugar da temperatura do corpo humana é entre $34^{\circ} \mathrm{C}$ até $40^{\circ} \mathrm{C}$, usou-se a temperatura entre $29^{\circ} \mathrm{C}$ até $87^{\circ} \mathrm{C}$. Para essa variação, se perturbação existir estará presente de forma clara nos resultados finais para o estudo de próton terapia.

\subsection{SIMULAÇÃO DOS EVENTOS}

Utilizando o hadrontherapy [2] do GEANT4 que está nos exemplos avançados, foram realizouse mudanças na geometria do detector da Fig 2 por um copo de água da Fig 3.

Figura 2: Representação do "hadrontherapy" do Geant4.

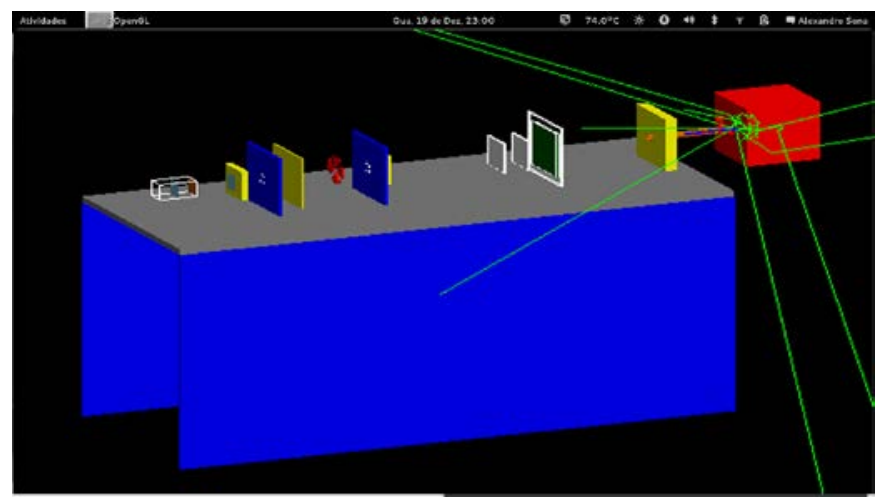


Figura 3: Esquema do alvo de água acoplado ao "hadrontherapy" do Geant4.

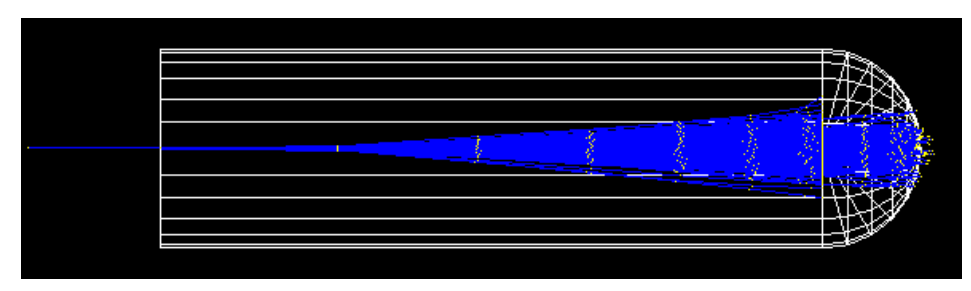

Possibilitando simular cada evento com suas características definidas previamente pelo processo de analise $2^{\mathrm{k}}$ sendo realizado os ensaios para cada um conjunto de parâmetros como apresentado na Tabela 1.

Com esta tabela fora calculada as interações dos fatores das seguintes formas, para a dose média nos volumes que houve deposição, são cinco fatores k=5 com variações de “-” para os valores menores e “+” para os valores maiores, então temos $2^{\mathrm{k}}=2^{5}=32$ formas de relacionar os cinco fatores, sendo (1) distância, (2) energia, (3) temperatura, (4) pressão, (5) densidade, (12) distância e energia, (13) distância e temperatura e assim por diante até relacionar todas as interações (12345): cinco efeitos individuas (1, 2, 3, 4 e 5); dez interações de dois fatores (12, 13, 14, 15, 23, 24, 25, 34, 35 e 45); dez interações de três fatores $(123,124,125,134,135,145$, 234, 235, 245 e 345); cinco interações de quatro fatores (1234, 1235, 1245, 1345 e 2345) e uma interação de cinco fatores (12345).

Onde aplicou-se a densidade e pressão que demonstraria maior significância nos resultados. Pode ser observado na Tabela 1, que do ensaio 01 ao 16 fora mantido a densidade fixa no valor baixo (-) de $1.01 \mathrm{~g} / \mathrm{cm}^{3}$ e foram variados os outros fatores. Os cinco fatores possuem 16 ensaios fixos em alta (+) e 16 em baixa (-), totalizando 32 ensaios.

Para o caso de $2^{k}$, não podemos estimar o erro experimental da forma que deveria ser feito para todos os fatores, uma vez que é uma simulação que fornece o erro associado que é o inverso do número de eventos simulados. Sendo o número de eventos da simulação da ordem de $10^{7}$ reduz o erro da simulação. 
Nota-se que quão maior é o $k$, mais efeitos de interações surgem, com cinco fatores temos vinte e sete (27) interações entre dois, três, quatro e cinco fatores. À medida que o número de fatores aumenta, o modelo matemático também aumenta, incluindo cada vez mais termos. Com $k$ fatores, o modelo teria de continuar até a interação de todos os $k$ fatores, como a expansão de uma função numa série de potências.

\section{RESULTADOS E DISCUSSÕES}

As respostas obtidas pelo estudo para o comportamento da dose por prótons no ponto de formação do Pico de Bragg [3] em água para diferentes variáveis de entrada.

Gerando a matriz transposta dos contrastes das interações dos fatores, expresso na Tabela 1, para calcular os efeitos das interações. Com a matriz transposta e efetuando o produto com a matriz coluna das médias de todos os ensaios e dividindo por $2^{\mathrm{k}-1}=16$ para todos valores e $2^{\mathrm{k}}=32$ para o valor da média na primeira linha da matriz transposta, como mostrar na Tabela 2. Obtivimos as seguintes relevância para os efeitos como apresentado na Tabela 3.

Relacionando a distribuição de efeitos com uma distribuição normal para ter-se $90 \%$ dos valores, constrói-se a relação da calda de distribuição normal padrão com a distribuição dos efeitos. Com o estudo estatístico, Projeto Fatorial $2^{k}$, o erro-padrão foi de aproximadamente 3,9 ficando um número reduzido de fatores que influenciam a deposição de energia de um feixe de prótons. 
Tabela 1: Dados da simulação, variando os cinco parâmetros com total de 32 ensaios.

\begin{tabular}{|c|c|c|c|c|c|c|}
\hline & distância & energia & temperatura & pressão & densidade & $\begin{array}{r}\text { Média } \\
\text { Dose(MeV/g) } \\
\end{array}$ \\
\hline 1 & + & - & - & - & - & 263,8700 \\
\hline 2 & - & - & - & - & - & 273,4663 \\
\hline 3 & + & + & - & - & - & 820,4794 \\
\hline 4 & - & + & - & - & - & 820,5504 \\
\hline 5 & + & - & + & - & - & 263,8700 \\
\hline 6 & - & - & + & - & - & 273,4663 \\
\hline 7 & + & + & + & - & - & 820,4794 \\
\hline 8 & - & + & + & - & - & 820,5504 \\
\hline 9 & + & - & - & + & - & 263,8700 \\
\hline 10 & - & - & - & + & - & 273,4663 \\
\hline 11 & + & + & - & + & - & 820,4794 \\
\hline 12 & - & + & - & + & - & 820,5504 \\
\hline 13 & + & - & + & + & - & 263,8700 \\
\hline 14 & - & - & + & + & - & 273,4663 \\
\hline 15 & + & + & + & + & - & 820,4794 \\
\hline 16 & - & + & + & + & - & 820,5504 \\
\hline 17 & + & - & - & - & + & 88,0099 \\
\hline 18 & - & - & - & - & + & 91,1430 \\
\hline 19 & + & + & - & - & + & 284,7273 \\
\hline 20 & - & + & - & - & + & 275,3094 \\
\hline 21 & + & - & + & - & + & 88,0099 \\
\hline 22 & - & - & + & - & + & 91,1430 \\
\hline 23 & + & + & + & - & + & 284,7273 \\
\hline 24 & - & + & + & - & + & 275,3094 \\
\hline 25 & + & - & - & + & + & 88,0099 \\
\hline 26 & - & - & - & + & + & 91,1430 \\
\hline 27 & + & + & - & + & + & 274,7118 \\
\hline 28 & - & + & - & + & + & 275,3094 \\
\hline 29 & + & - & + & + & + & 88,0099 \\
\hline 30 & - & - & + & + & + & 91,1430 \\
\hline 31 & + & + & + & + & + & 274,7118 \\
\hline 32 & - & + & + & + & + & 275,3094 \\
\hline
\end{tabular}

\begin{tabular}{lcc} 
& + & - \\
\hline distância & $300 \mathrm{~cm}$ & $100 \mathrm{~cm}$ \\
\hline energia & $54 \mathrm{MeV}$ & $18 \mathrm{MeV}$ \\
\hline temperatura & $87^{\circ} \mathrm{C}$ & $29^{\circ} \mathrm{C}$ \\
\hline pressão & $3 \mathrm{~atm}$ & $1 \mathrm{~atm}$ \\
\hline densidade & $3.01 \mathrm{~g} / \mathrm{cm} 3$ & $1.01 \mathrm{~g} / \mathrm{cm} 3$ \\
\hline
\end{tabular}


Tabela 2: Produto da matriz transposta dos efeitos das interações pela matriz linha das médias de dose.

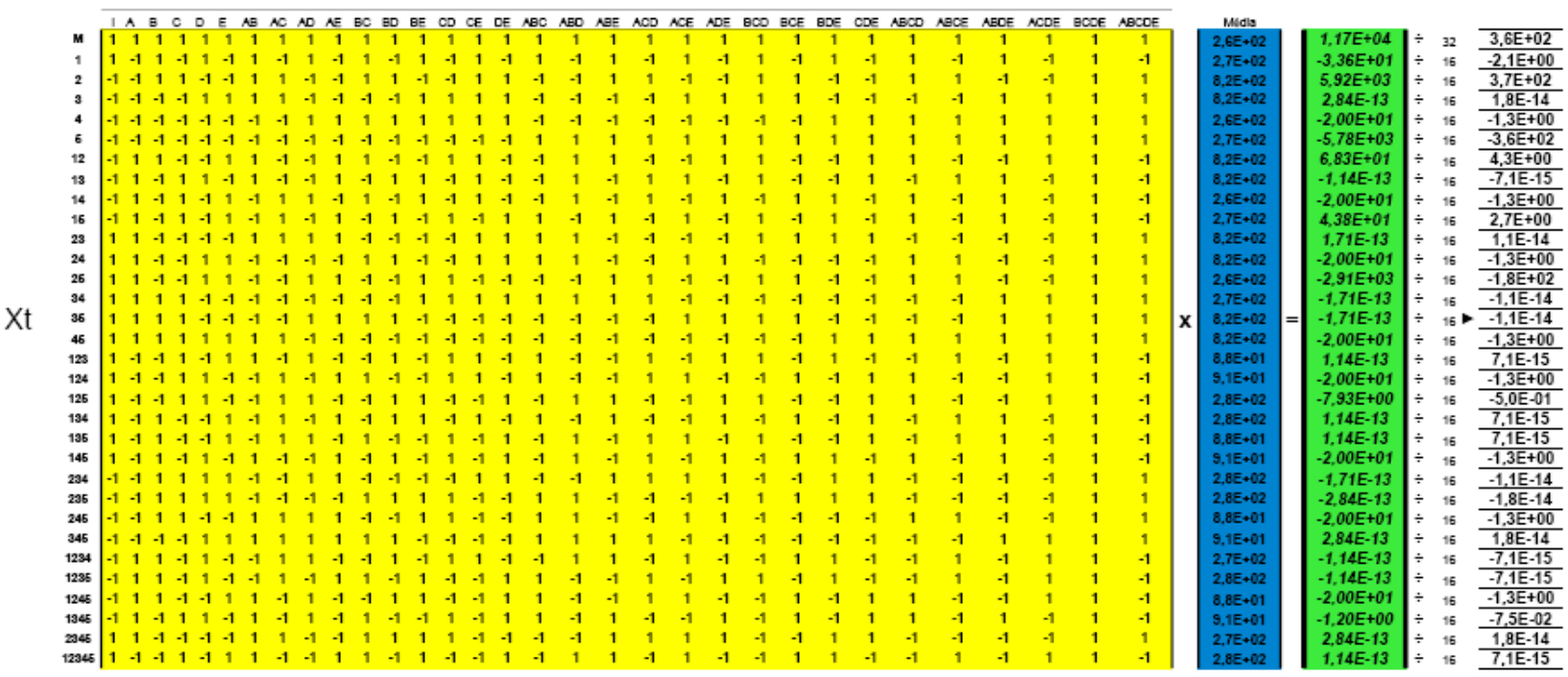

Tabela 3: Efeito sem interação, e com de dois, três, quatro e cinco interações.

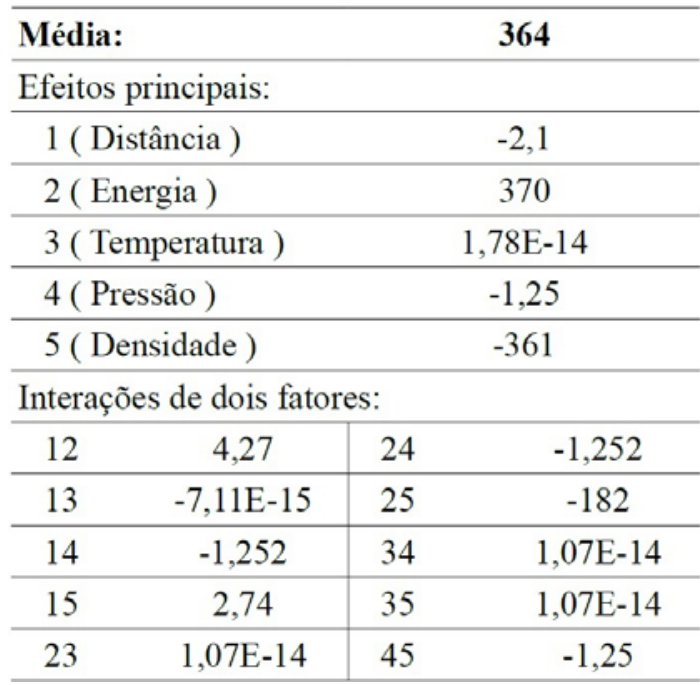

\begin{tabular}{|c|c|c|c|}
\hline \multicolumn{4}{|c|}{ Interações de três fatores: } \\
\hline 123 & $7,11 \mathrm{E}-15$ & 145 & $-1,252$ \\
\hline 124 & $-1,252$ & 234 & $1,07 \mathrm{E}-14$ \\
\hline 125 & $-4,95 \mathrm{E}-1$ & 235 & $-1,78 \mathrm{E}-14$ \\
\hline 134 & $7,11 \mathrm{E}-15$ & 245 & $-1,252$ \\
\hline 135 & $7,11 \mathrm{E}-15$ & 345 & $1,78 \mathrm{E}-14$ \\
\hline \multicolumn{4}{|c|}{ Interações de quatro fatores: } \\
\hline 1234 & $7,11 \mathrm{E}-15$ & 1345 & $-7,47 \mathrm{E}-2$ \\
\hline 1235 & $7,11 \mathrm{E}-15$ & 2345 & $1,78 \mathrm{E}-2$ \\
\hline 1245 & $-1,252$ & & \\
\hline \multicolumn{4}{|c|}{$\begin{array}{l}\text { Interações de cinco } \\
\text { fatores: }\end{array}$} \\
\hline 12345 & & & -5 \\
\hline
\end{tabular}

Na Fig 4 é apresentado o gráfico a partir das Tabelas 3 e distribuição normal. O Fator 1, tem relevância, porém muito menor que os fatores 2 e 5 . Já os fatores 3 e 4, estão dentro do erropadrão, mostrando que 3 e 4, são irrelevantes para o estudo da dose. 
Figura 4: Distribuição normal dos efeitos das variáveis e suas interações.

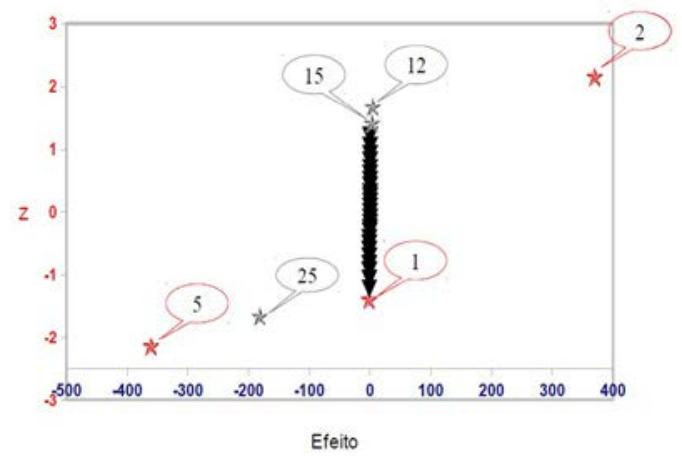

\section{CONCLUSÕES}

A dose absorvida deverá ter um comportamento dependente da energia do feixe e densidade do material e sua deposição em função da profundidade apresente um pico de bragg bem definido.

Os fatores experimentais que mais influenciam os resultados de deposição de dose estão relacionados com energia do feixe, densidade do alvo e número atômico do alvo.

Como parte integrante de um estudo da dose por feixe de prótons em tecido equivalente, tem como perspectiva: Realizar estudo de dosimetria de uma sala com uma fonte de prótons; Desenvolver estudo para utilizar um feixe de prótons, não só para produção de radiofármaco, bem com para tratamento e produção de nêutrons; Determinar os procedimentos para tratamento de tumores com um feixe de prótons.

\section{AGRADECIMENTO}

Agradeço aos colegas acadêmicos do Departamento de Energia Nuclear da Universidade Federal de Pernambuco (DEN/UFPE) e Centro Regional de Ciências Nucleares do Nordeste da Comissão Nacional de Energia Nuclear (CRCN-NE/CNEN) e ao CNPq pelo apoio financeiro e intelectual. 


\section{REFERÊNCIAS}

1. MONTGOMERY, D. C., Design and Analysis of Experiments, John Wiley \& Sons, 2001.

2. AGOSTINELLI, S. “Geant4 - a simulation toolkit”. Nuclear Instruments and Methods in Physics Research, v. 506, p. 256-303, 2003.

3. PAGANETTI, H. Proton Therapy Physics, CRC Press, 2011. 